dauernden, of $t$ unterbrochenen, aber immer wieder auflebenden Hebungstendenz beherrscht waren. Meistens werden solche Elevationen des Meeresbodens von aneinander gereihten Inseln, Untiefen und Bänken gebildet, die in der Tiefe $z \mathfrak{u}$ einer submarinen Schwelle sich zusammenschließen. Als Beispiele wären aus der zusammenfassenden Darstellung von ARLDt (1917) die nordatlantische und die lemurische Landbrücke zu nennen. Sie sind in fazieller Beziehung durch das Vorkommen von Seichtwassermollusken, anderseits durch seismische Äußerungen gekennzeichnet ${ }^{1}$ ). Wenn die dauernde aber an Intensität wechselnde Hebungstendenz sich zeitweise steigert, so wird es möglich, daß eine submarine Elevation, sonst in Inseln und Bänke aufgelöst, in einem bestimmten, geologisch kurz bemessenen Zeitabschnitt als eine relativ schmale, kontinuierliche Landbrücke emportaucht, um für kurze Zeit den interkontinentalen Organismenaustausch zu ermöglichen, bald aber durch neuerliche Auflösung in einen Inselbogen wieder zu einer unüberwindlichen Schranke zu werden.

\title{
Zu A. Kampfraths Aufsatz über die Geländestufen und Geländegräben der Umgebung von Dresden.
}

\author{
Von Kurt Pietzsch (Leipzig).
}

Auf den topographischen Karten 1:25000 (MeBtischblättern), die auch die Grundlage der geologischen Spezialkarten bilden, werden Steilränder oder Geländestufen mit einer besonderen Signatur (-gekennzeichnet, sobald sie eine zu starke Neigung oder eine zu geringe Höhe besitzen, als daß die gewöhnliche Darstellungsart durch Höhenkurven möglich ist. Zwei gegeneinander gekehrte solche Zeichen (=-i) stellen einen Graben im Gelände oder einen Hohlweg dar. Derartige "Geländestufen " und "-gräben " sind mit der angegebenen Signatur auf den neueren Meßtischblättern vollständiger verzeichnet als auf den älteren. Mit ihrer Entstehung beschäftigt sich ein Aufsatz von A. Kamprrath im 1. Heft dieses Jahrgangs der Geologischen Rundschau.

A. KAMPFRATH hat alle diejenigen )Geländestufen \& und »-gräben*, die auf den Mebtischblättern der Dresdener Gegend verzeichnet sind, und die nicht waugenscheinlich künstliche Straßen- und Eisenbahneinschnitte und -böschungen " darstellen, auf seiner Übersichtskarte, Taf. I, zusammengestellt, ohne ihre Entstehung in jedem einzelnen Falle

1) ARLDT (1917), S. 86, 164. 
weiter nachzuprüfen. Er behauptet, die Geländestufen seien wzutage tretende Rutsch- und Verwerfungsflächen « und die Geländegräben ursprünglich "klaffende Spalten "; und er erblickt in diesen Gebilden »die Zeugen eines vorgeschichtlichen heftigen Erdbebens «. Diese Behauptung wird durch keinerlei unmittelbare Beobachtung über Schichtenverschiebungen an den "Stufen " oder das Vorhandensein von Spalten am Grunde der Hohlwege gestützt und muß als durchaus verfehlt bezeichnet werden. Nach der in Geologenkreisen bisher wohl allgemein verbreiteten Auffassung und nach meinen Erfahrungen, die gewiß auch jeder andere Aufnahmsgeolog in anderen Gegenden in ähnlicher Weise zu machen reichlich Gelegenheit gehabt haben wird, erklärt sich die Entstehung der "Geländestufen und -gräben « KAMPFRATHs viel zwangloser und ohne Zuhilfenahme hypothetischer vorgeschichtlicher Ereignisse.

Die Geländestufen finden sich zu einem kleinen Teile im Bereich der Talböden und stellen hier alte Erosionsränder dar, die aber durch Menschenhand vielfache Veränderungen (vor allem eine Befestigung) erfahren haben. Hierzu gehört z. B. eine von Kampfrath mit genannte, von einem Fußweg begleitete Stufe zwischen der Fröbelstraße und dem Weißeritzufer im südwestlichen Teile von Dresden. Wie die 2. Auflage des Blattes Dresden der geologischen Karte zeigt, stellt diese Stufe den Rand der jungdiluvialen Weißeritzterrasse (d3w) gegen das Taldiluvium dar. Auch im letzteren selbst sind ähnliche Stufen vorhanden. Mit Verschiebung der Erdschichten im Gefolge eines Erdbebens haben alle diese Steilränder nicht das Geringste zu tun.

In ihrer großen Mehrzahl sind die Geländestufen KampFratis aber nicht auf den Talböden, sondern an den Gehängen und auf den Hochflächen gelegen und unter wesentlicher Nitwirkung des Menschen entstanden. Abgesehen von den z. T. verstürzten Rändern alter Steinbrüche, Sandgruben, Lehmgruben u. dgl. sind die mit der oben gekennzeichneten Signatur angegebenen Geländestufen eine Folge der Ausnutzung des Bodens für die Landwirtschaft. Es ist jedem Landmann bekannt, daß bei starken Regengïssen auf geneigten Feldoberflächen eine beträchtliche Abschwemmung von den höher gelegenen Feldteilen erfolgt (die sich z. B. bei unzweckmäßiger Anlage der Kartoffeldämme sehr unangenehm bemerkbar machen kann), und daß gleichzeitig eine Verschlämmung der tiefer gelegenen Teile des Feldes eintritt. Man hat deshalb von altersher solche ungünstigen Neigungsverhältnisse der Feldflächen auszugleichen gesucht. Bei Flurstücken, die annähernd parallel den Höhenkurven, also ungefähr horizontal am Hange hin verlaufen, erreicht man dies, falls die Neigung nicht zu stark ist, schon beim Ackern, indem man in den höher gelegenen Teilen des Feldes die Flugschar kräftiger und tiefer eindringen läßt als in den tieferen. Bei alljährlicher Wiederholung dieses Verfahrens kommt in Verbindung mit dem allmählichen Abwärtswandern der oberflächlichen Erdschichten (Gekriech) eine Abtragung der höher gelegenen Feldteile 
und eine Aufhöhung der niedriger gelegenen zustande. Wird das Verfahren bei mehreren am Hange übereinander gelegenen Feldern durchgeführt, so ergibt sich eine gewisse Terrassierung des Hanges. Die einzelnen Felder müssen dann mit steilen Stufen aneinander grenzen, an deren Erhaltung die Besitzer der Felder natürlich großes Interesse haben, und auf denen sie möglichst eine dichte Grasnarbe zu erhalten suchen. Denn bei einer Zerstörung des Steilrandes würden nicht nur breite, unwirtschaftliche Hänge zwischen beiden Feldern entstehen, sondern es würde für die oben angrenzenden Felder die dauernde Gefahr der Abschwemmung und für die unteren diejenige der Versohlämmung in erhöhtem Maße eintreten. Infolge dieser Entstehung der Stufen firidet man anf den Teilen der Felder, die unten an die Stufen angrenzen, wo also eine Abtragung des Bodens stattgefunden hat, vielfach die sonst unter der oberflächlichen Deckschicht (meist Löß oder Lößlehm) verborgenen älteren Schichten (häufig altdiluviale Schotter) bloßgelegt, während nach KaMPFRATHs Annahme diese Schichten hier gerade abgesenkt sein müßten.

Die beschriebene Abstufung der Gehänge führt man natürlich nur soweit durch, als es nötig erscheint, oder als es sich bei der Feldbestellung ganz von selbst ergibt. Die Stufen begleiten daher die Feldgrenzen oft nur streckenweise. Wo die Feldflächen im allgemeinen durch gerade verlaufende, parallele Linien begrenzt werden, schneiden die Stufen die Höhenkurven natürlich unter den verschiedensten Winkeln. Wo die Flurstïcke gekrürnmte Flächen darstellen und horizontal am Hange verlaufen, wie es am Abhange einzelner, die Landschaft überragender Basaltkuppen, z. B. am Luchberg bei Dippoldiswalde, der Fall ist, verlaufen die Stufen häufiger parallel mit den Höhenkurven. - An intensiver kultivierten Gehängen, die seit langem für den Gartenbau und besonders den Weinbau ausgenutzt wurden, ist die Terrassierung gewöhnlich in verstärktem Maße und auch sorgfältiger durchgeführt als auf freien Feldflächen. Vielfach sind hier die. Stufen noch durch Steinpackungen oder durch Mauern gegen Zerstörung geschützt. Zu solchen künstlichen Terrassierungen gehören die von KAMPFrath zitierten Steilränder bei Gommern und Torna. Die von ihm beschriebene Verschiebung der Geländestufen bei Torna längs eines 》Querbruchs « rührt einfach daher, daß mit letzterem die Flargrenze zwischen Torna und Leubnitz zusammenfällt. Da die Flurstücke rechts und links von einer Flurgrenze nur selten gleich breit sind, so können bei künstlicher Abstufung des Hanges Geländestufen links und rechts der Flurgrenze natürlich nicht immer unmittelbar aufeinander stoßen; daher die verschiedene Zahl und Lage der "Stufen " und der "Querbruch (c.

Bei Flurstücken, die sich ungefähr senkrecht zum Verlauf der Höhenkurven erstrecken und die sich von einem. flach geneigten, über ein stärker geböschtes Stück nach einem wieder flacheren Gehängeteil hinziehen, oder die über eine Geländekuppe bzw. einen Höhenrücken 
hinweggehen, sucht man bisweilen eine Ausgleichung der verschieden starken oder verschieden gerichteten Neigungen dadurch zu erzielen, daß man den mittleren ungünstigeren Teil beim Ackern wabtreibt «. Wenn dann die Nachbarn nicht auch das gleiche Verfahren einschlagen, müssen natürlich an beiden Seiten des Flurstücks streckenweise Geländestufen entstehen, welche einen Teil des Feldes grabenartig versenkt erscheinen lassen.

Die mit der obengenannten Signatur auf den Karten angegebenen Geländegräben stellen teils alte Wassergxäben, vereinzelt auch alte, verlassene, natürliche Wasserläufe dar und finden sich dann vor allem auf den Hochflächen oder den Talböden. Zum größeren Teile sind es aber alte Hohlwege, die wohl meistens wegen des schlechten Zustandes und der schwierigen Unterhaltung der in ihnen verlaufenden Fahrstraßen verlassen worden sind. Werden solche Hohlwege nicht mehr befahren, sondern sich selbst überlassen, so verstürzen ihre Wände bald, und es bildet sich meist rasch ein V-förmiger Querschnitt bei den ehemals U-förmigen Hohlwegen heraus, so dabie jetzt gewöhnlich nur noch als Fußwege benutzt werden können. Die Hohlwege selbst mögen ursprünglich z. T. absichtlich angelegt worden sein, meistens entstehen sie aber an den Gehängen schon von selbst infolge der hier besonders starken Beanspruchung der Fahrbahn beim Bremsen des Wagens und infolge der am Gehänge stärker wirkenden Abschwemmung. Abfließende Wassermassen stärkerer Gewittergüsse, die auf die Hochfläche auftreffen, reißen oft ganz ansehnliche Rinnen in die am Hange herabführenden Wege und vertiefen dabei einesteils die Hohlwege, anderenteils zwingen sie gelegentlich zur Preisgabe des Weges überhaupt. Man findet deshalb nicht selten neue, besser gebaute Wege oben neben den alten Hohlwegen entlang führen. In lockerem Katerial von geeigneter Beschaffenheit, wie im Löß und im Gehängelehm, entstehen natürlich Hohlwege leichter als im festen Gestein, obwohl sie auch in diesem gelegentlich vorkommen. - Mit ursprünglich klaffenden Spalten haben also die Hohlwege der Dresdener Gegend nicht das Geringste zu tun. Auf ihrem Boden trifft man tatsächlich an manchen Stellen das unter der oberflächlichen Deckschicht (LöB, Lößlehm usw.) anstehende feste Gestein (z. B. Pläner), bis auf welches sich der Weg eingetieft hat, wie in einem vom Ortsteil Weidental nach Obergorbitz führenden Hohlweg.

So viel über die Entstehung der "Geländestufen und -gräben ", die von jedem nachgeprüft werden kann, der Gelegenheit hat, die Beschaffenheit und Veränderung des Erdbodens während der verschiedenen Jahreszeiten und bei verschiedener Witterung genauer zu beobachten. Die Behauptungen Kamprraths über die Entstehung dieser Gebilde sind durch keine tatsächlichen Beobachtungen gestützt und mässen aufs Entschiedenste zurückgewiesen werden.

Gleiches gilt von Kamprraths Behauptung, daß die Stufen am linken Elbtalgehänge der sichtbare Ausdruck einer hier vorhandenen 
größeren Verwerfung seien, an der die Absenkung der "Elbtalscholle" erfolgte. Gewißlich fordern die Lagerungsverhältnisse der Kreide hier die Annahme einer Störung, worauf ich in der 2. Auflage der Erläuterungen zu den Blättern Pirna und Kreischa der geologischen Karte bereits hinwies, aber die Terrassierungen des Gehänges bei Torna, Gommern usw. haben nichts mit dieser Verwerfung zu tun. Sie kann auch nicht durch die verschiedene Höhenlage der Auflagerungsfläche der Kreide auf das Grundgebirge erwiesen werden, die man am Gehänge bei der Pechhütte bei Großsedlitz und in einiger Entfernung in einer Tiefbohrung in Hoeschs Zellstofffabrik bei Pirna festgestellt hat. Denn bei der Pechhütte liegt Labiatuspläner, in der Bohrung aber die Carinatenstufe auf dem Granit. Die vorcenomane Granitoberfläche war bekanntlich keine tischebene "Abrasionsfläche ", sondern besaß recht erhebliche Unebenheiten; als Vergleichsfläche bei der Feststellung einer Sprunghöhe, wie es KampraAtH tut, kann sie daher selbstverständlich nicht verwendet werden.

Auf schwachen Füßen steht ferner KampFiratrs Vermutung, daß die breiten Talauen der Seidewitz und Gottleuba Grabenversenkungen seien. KampFrath benutzt zum Beweis eine Bohrung, die i.J. 1888 in der neuen Kaserne in Pirna gestoßen wurde, und errechnet eine Absenkung der Schichten unter der Gottleubaaue um $29 \mathrm{~m}$. Dazu ist aber zu bemerken, daB dies nux unter der Voraussetzung Geltung haben kann, daß der »oben tonig zersetzte Pläner ", welcher im Bohrloche unter dem Alluvium angetroffen wurde, dem Brongniartipläner ( $t 2 p$ ) der Talgehänge entspricht. Diese Annahme kann angesichts der sonst nur 18,5 m betragenden Mächtigkeit des unteren Grünsandstein- und Nergelkomplexes der Brongniartistufe kaum zutreffen. Viel wahrscheinlicher ist es, daß der "Pläner " des Bohrlochs nur eine harte Mergelbank innerhalb des Komplexes der Grünsandsteine darstellt. Überdies ist die von KaMPFRATH zum Vergleich der Höhenlage der Kreideschichten benutzte Grenze zwischen Labiatus- und Carinatenstufe innerhalb des Bohrlochs nicht genau zu bestimmen. Wie man sich in der von KAMPFRATH an anderer Stelle zitierten, ihm also nicht unbekannten 2. Auflage der Erläuterungen zu Blatt Pirna auf S. 156 überzeugen mag, habe ich daher bei diesem Bohrloch die Lage dieser Grenze unentschieden gelassen. Sie kann jedenfalls nicht so gelegen sein, wie es in der 1. Auflage angenommen und von KaMPFrate seiner Berechnung zugrunde gelegt wurde, weil die $12,60 \mathrm{~m}$ groben, lockeren Quadersandsteine lediglich der unteren Abteilung der Carinatenstufe entsprechen; die höhere Abteilung wird fast durchweg von feinkörnigen, $z$. T. tonigen und kalkigen Sandsteinen (Plänersandstein) gebildet und ist naturgemäß dort von der Labiatusstufe nicht zu trennen, wo diese, wie in der Gegend von Pirna, mit ähnlicher Gesteinsausbildung entwickelt ist.

Auch Kamperaths Bemerkungen ïber die tektonischen Vorgänge, welche bei der Bildung des Dresdener Elbtalkessels mitgewirkt haben, 
können einer Kritik nur schlecht standhalten. Die nach Südwesten gerichtete Verschiebung des „Radeberger Tafelstücks der Lausitzer Granitplatte « längs der Linie GroBgraupe-Dittersbach-Harthau-Burckau gegen das südöstlich anstoßende wStolpener Tafelstück «, sowie das Hinüberschieben des ersteren auf die vorher schon eingetretene Lausitzer Uberschiebung und sein Hinaufwandern auf die "Elbauenscholle", und endlich der Zusammenhang der Bachläufe mit diesen Störungen, sind durch nichts bewiesene Vermutungen, wie sie einem wohl gelegentlich beim Betrachten der geologischen Karte kommen mögen, die man sich aber zu veröffentlichen hütet, so lange keine exakten Beweise erbracht werden können.

Anlaß zu Widerspruch bietet schließlich Kampratirs Geschichte des Elbtals, die er auf S. 29 auch tabellarisch zusammenfaßt. Mit welchem Recht z. B. die altdiluvialen Schotter $\left(\mathrm{d}_{1}, \mathrm{~d}_{1} \mathrm{e}, \mathrm{d}_{1} \varepsilon\right)$ durchweg einer älteren Eiszeit angehören sollen, als der Geschiebelehm (d2), ist nicht ersichtlich. Bezüglich der Diluvialbildungen des Elbtales sei vorläufig auf die 2. Auflage der Erläuterungen zu den Blättern Pirna, Kreischa, Dresden und Pillnitz, sowie auf meine Arbeit über den spflanzenführenden Glazialton von Luga bei Dresden und die Gliederung des Elbtaldiluviums « (Sitzb. d. Naturf. Ges. Leipzig 1915, S. 21-54) verwiesen.

Auf alle Behauptungen KaMpFraTHs im einzelnen einzugehen, würde hier viel zu weit führen. Ich muß mich vielmehr angesichts seiner offen zutage liegenden Irrtümer mit obigen Einwendungen begnügen. Bloße Vermutungen oder Ansichten, wie sie A. Kamprrath ohne Begründung durch Beobachtungstatsachen zahlreich vorbringt, können in der Literatur nur Verwirrung anstiften und hemmen die wissenschaftliche Erkenntnis statt sie zu fördern. 